\title{
Research on Classification Environment Technology Diffusion of Occupational Tobacco Growers
}

\author{
Feng Wang ${ }^{1, a}$, Xiangdan $\mathrm{Hu}^{1, \mathrm{~b}}$, Jun $\mathrm{Liu}^{2, \mathrm{c}^{*}}$ \\ ${ }^{1}$ Branch of Tobacco Company in Southwest Guizhou Province, Xingyi 562400 \\ ${ }^{2}$ Guizhou University of Finance and Economics, Guiyang 550025 \\ aemail: 479095320@qq.com, bemail: 5267865@qq.com, email: liujunsucceed@163.com
}

\begin{abstract}
Key words: Occupational Tobacco Growers; Environment Technology; Technology Diffusion Model

Abstract: With the technology diffusion model, time path function is deduced. Through the depicting of function graphic, we can find it is S-shaped curve. The results show that the internal and external factor of different parts, and the differences of the total number of "potential adopters" lead to the differences of time path function of classification environment technology diffusion of occupational tobacco growers in different areas, and the differences in different leaf tobacco production areas when marketing the technology.
\end{abstract}

\section{Introduction}

In 2013, the State Tobacco Bureau put forward that the key of tobacco industry's sustainable development is to solve the problem of " who is responsible for the cultivation of tobacco," cultivate occupational tobacco growers, professional services team and workers of tobacco leaf industry, accelerate three separate of professional services in land ownership, operation and management ${ }^{[1]}$. So what kind of farmers are occupational tobacco growers, how many types are there, how to assess them, what is the environment standard, what is the difference between current farmers and them? Combined with the situation of mountain, we make discussion and applied research, hoping to provide reference for cultivation of occupational tobacco growers.

Kenneth Hacker and Jan van Dijk (2013) analyzed the statistical data of tobacco growing farm between United States and Netherlands from 2004 to $2010^{[2]}$. Jos De Haan( 2004 ) put forward the gap of classification environment technology diffusion of occupational tobacco growers and established "causal feedback loop" model which illustrate the process of classification environment technology diffusion of occupational tobacco growers from the perspective of causal feedback ${ }^{[3]}$. Jayajit Chakraborty and M. Martin Bosman(2005), according to statistical data of income and knowledge level of tobacco growing area in several states of United States, combined with Lorenz Curve and Gini Coefficient, presented the development process of classification environment technology diffusion of occupational tobacco growers ${ }^{[4]}$. Tim Turpin and Russel Cooper (2005), researched the differences of classification environment technology diffusion of occupational tobacco growers between developing countries ${ }^{[5]}$. 


\section{Classification Environment Technology Diffusion Model of Occupational Tobacco Growers}

\section{Basic Assumptions and Variables}

Before establish the model, combined with the characteristics of classification environment technology diffusion ${ }^{[6]}$, this paper gives the following basic assumptions and description of relevant variables.

The total number of potential adopters is $N(N>0$, It is generally equal to the region's total population). At the $t$ moment, there are $y(t)$ "potential adopters" who have adopted classification environment technology of occupational tobacco growers, and there are $\{N-y(t)\}$ who have not adopted the technology .

\section{Time Path Function of Classification Environment Technology Diffusion of Occupational} Tobacco Growers

\section{Derivation of Time Path Function}

Starting from $t$ time, within a time interval $\Delta t$, the existing people who not adopt is affected by the external and internal information sources.

Within a time interval $\Delta t$, the increasing number of "adopted" caused by external information sources is $\Delta y(t)^{*}=\alpha\{N-y(t)\} \Delta t$.

If the effect that "not adopters" were told by external sources or internal information is equivalent, within a time interval $\Delta t$, the increased number of "adopted" is

$$
\left.\Delta y(t)=\Delta y(t)^{*}+\Delta y(t)^{* *}=\{\alpha+\beta y(t)\} N-y(t)\right\} \Delta t
$$

Both sides of equation (1) are divided by $\Delta t$, and take the limit, differential equation is obtained.

$$
\left.\frac{d y(t)}{d t}=\{\alpha+\beta y(t)\} N-y(t)\right\}
$$

With equivalent transformation and integration, equation (2) becomes

$$
y(t)=\frac{C N \exp [(\alpha+\beta N) t]-\alpha}{\beta+C \exp [(\alpha+\beta N) t]}
$$

\section{Graph of Time Path Function}

Equation (3) is elementary function, so it must be continuous. Its first order derivative is

$$
Y^{\prime}(t)=\frac{1}{N}\left\{\frac{T \beta+\alpha}{N-T} \exp [(\alpha+\beta N) t](\alpha+\beta N)^{2}\right\}\left\{\beta+\frac{T \beta+\alpha}{N-T} \exp [(\alpha+\beta N) t]\right\}^{-2}
$$

Similarly, the first order derivative of Function $(4)$ is

$$
Y^{\prime \prime}(t)=\left\{(\alpha+\beta N)-2 \frac{C(\alpha+\beta N) \exp [(\alpha+\beta N) t]}{\beta+C \exp [(\alpha+\beta N) t]}\right\} Y^{\prime}(t)
$$


The equation (4) is substituted into equation (5), we can get (6) $Y^{\prime \prime}(t)=\left\{(\alpha+\beta N)-\frac{2 C(\alpha+\beta N) \exp [(\alpha+\beta N) t]}{\beta+C \exp [(\alpha+\beta N) t]}\right\}\left\{\frac{C}{N} \exp [(\alpha+\beta N) t](\alpha+\beta N)^{2}\right\}\{\beta+C \exp [(\alpha+\beta N) t]\}^{-2}$

Order $Y^{\prime \prime}(t)=0$, we can get turning point of equation (6), with hypothesis 2 , hypothesis 3 and hypothesis 4 , we know $Y^{\prime}(t)>0$. Then $Y^{\prime \prime}(t)=0$ is equivalent to

$$
(\alpha+\beta N)-2 \frac{C(\alpha+\beta N) \exp [(\alpha+\beta N) t]}{\beta+C \exp [(\alpha+\beta N) t]}=0
$$

The solutions of equation (6) is $t=t_{\text {inf lexion }}=\frac{\ln \frac{\beta}{C}}{(\alpha+\beta N)}$, Point $\left(t_{\text {inf lexion }}, Y\left(t_{\text {inf lexion }}\right)\right)$ is turning point of equation (7).

With turning point, we can get concave convex interval of equation (7). Equation (7) is elementary function, we can get its first order derivative is (12) $Y^{\prime \prime \prime}(t)=\phi^{4} C \exp (\phi t)[\beta+C \exp (\phi t)]^{-3}\left\{\beta-2 C \exp (\phi t)-3 C \exp (\phi t)[\beta-C \exp (\phi t)] \cdot[\beta+C \exp (\phi t)]^{-1}\right\}$

And, $\phi=\alpha+\beta N$.

With the judging theorem of concave convex, and $Y^{\prime \prime}(0)=\left(\frac{\alpha \beta+\beta^{2} N}{\beta+C}\right)\left(\frac{C(\alpha+\beta N)^{2}}{(\beta+C)^{2}}\right)>0$, on interval $t \in\left[0, t_{\text {inf lexion }}\right), Y^{\prime \prime \prime}(t)>0$, namely, $Y^{\prime \prime}(t)$ is monotone increasing on interval $t \in\left[0, t_{\text {inf lexion }}\right)$. So $Y^{\prime \prime}(t)>0$ on interval $t \in\left[0, t_{\text {inf lexion }}\right)$, function ( 7$)$ is concave.

In summary, the formula (7) can be made as shown in Figure 1, which is a S shaped curve. The proportion $Y(t)$ of "adopted" accounted for "potential adopters" is actually determined by four parameters $\alpha, \beta, N, T$. The graph can reflect this changes that classification environment technology diffusion of occupational tobacco growers presents $\mathrm{S}$ shape curve growth model over time.

\section{Conclusion}

First, the differences of external factors in different regions lead to different parameters $\alpha$; second, the differences of internal factors in different regions lead to different parameters $\beta$. In addition, the total number $\mathrm{N}$ of "potential adopters" and initially number $T$ of users in different regions are generally different. Therefore, due to different parameters $\alpha, \beta, N, T$ in different regions, time path function $Y(t)$ is different. At the same time point, difference of function values show a difference between two regions. Over time, this difference will gradually expand, stable down and 
gradually reduce. This three-stage process of evolution is a universal regularity. This conclusions can explain the difference of classification environment technology diffusion of occupational tobacco growers between different tobacco growing areas and different tobacco farm.

\section{Reference}

[1] Zhao Qiangshe. Research on cultivation path of professional farmers[J]. Journal of Socialist Theory Guide, 2009(3):56-58.

[2] Kenneth Hacker, Jan van Dijk. The Digital Divide as a Complex and Dynamic Phenomenon [J].The Information Society, 2003, (19): 315-326.

[3] Jos De Haan.A Multifaceted Dynamic Model of the Digital Divide [J]. IT\& Society, 2004, 1(7): 66-88.

[4] Jayajit Chakraborty, M. Martin Bosman. Measuring the Digital Divide in the United States: Race, Income, and Personal Computer Ownership [J]. The Professional Geographer, 2005, 57(3): 395-410.

[5] Tim Turpin, Russel Cooper. Technology, Adaptation, and Public Policy in Developing Countries: the "ins and outs" of the Digital Divide[J]. Essay Review, 2005, (5): 419-427.

[6] Liang Dan, Research progress of technology diffusion [J]. Scientific research management, 2005, 26 (4): 29-34. 